\title{
METHODS OF SCORING LINKAGE DATA GIVING THE SIMULTANEOUS SEGREGATION OF THREE FACTORS
}

\author{
C. RADHAKRISHNA RAO
}

King's College, Cambridge and Presidency College, Calcutta

\section{CONTENTS}

Received 28.v.47

1. Introduction . . . . . . . . 37

2. Theoretical Aspects of the Method of Scoring and Notations . 38

3. Scoring of Data from Back Crosses and Intercrosses . . . $\quad 40$

4. Maximum Likelihood Estimates of the Recombination Fractions 44

5. Maximum Likelihood Estimates Subject to Kosambi's Formula . 45

6. An Illustrative Example . . . . . . . . 46

7. The Increase in Precision by the Use of Efficient Scores . . 51

8. Summary . . . . . . . . . . 57

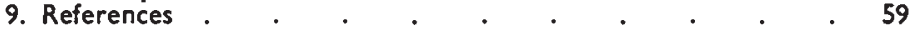

\section{INTRODUCTION}

When estimating the values of a number of recombination fractions for various segments of a chromosome, from linkage data, it is often the case that more than one body of data is available. The data may relate to various sources, different types of crosses and consist of parts supplying information for one or more of the segments. In such cases, it is, obviously, desirable to combine the data in order to make joint estimation of the parameters. The estimates, thus obtained, are the most efficient when different parts of the data are homogeneous which can, however, be directly verified by testing whether the estimates closely fit in with the various parts of the data.

Fisher (1935) put forward a method of scoring which seems fitted for general use where combination of data, tests of homogeneity and pooled estimates are considered. This method consists of replacing each body of data by appropriate scores and information which are directly used in arriving at pooled estimates and tests of homogeneity. In a paper Fisher $\left(194^{6}\right)$ used this method of scoring in estimating three recombination fractions (arising out of two consecutive segments and subject to Kosambi's (I944) restriction) from data which in parts supply information only for the individual segments. If the data giving the simultaneous segregation of three or more factors are available then two questions arise. What is the most appropriate method of scoring such data and how does it compare with the alternative method of scoring for each recombination fraction by considering the classification with respect to the two relevant factors only and ignoring the rest? The latter method of scoring may be called the individual segment method. 
When the simultaneous segregation of more than two factors has been recorded, the scores obtained by the individual segment method are less efficient in the sense in which Fisher (1935) defines efficient scores. The estimates to which they lead differ from the maximum likelihood estimates and hence are less efficient than the best estimates. The purpose of this article is to study these problems in the simplest case of scoring data on three factors arranged in eight phenotypical classes.

Firstly, a method appropriate and efficient for such data has been developed for the estimation of recombination fractions in the two different situations, viz. (I) when they are taken as free parameters and (2) when they conform to Kosambi's formula. Secondly, the relative efficiencies of the estimates in each of the two cases obtained by the individual segment method as compared with the above method have been calculated in a particular case to measure the loss in efficiency due to the individual segment method. The method of efficient scores involves a slightly heavier computational procedure and, though undoubtedly more efficient, can be recommended in practice only when even a small gain in efficiency is of considerable importance.

\section{THEORETICAL ASPECTS OF THE METHOD OF SCORING AND NOTATIONS}

Let there be $k$ sets of data, each part supplying information on one or more of a set of $p$ unknown parameters $\theta_{1}, \theta_{2}, \ldots, \theta_{p}$. If $\mathrm{L}_{1}, \mathrm{~L}_{2}, \ldots \mathrm{L}_{k}$ represent the probability densities of the observations corresponding to the $k$ sets of data then $\mathrm{L}$, the likelihood of the parameters, is defined by the product,

$$
\mathrm{L}=\mathrm{L}_{1} \mathrm{~L}_{2} \ldots \mathrm{L}_{k}
$$

The best estimates of the parameters are those values of $\theta_{1}, \ldots, \theta_{p}$ which maximise the above expression. A formal proof of this statement is given elsewhere (Rao, 1947). For convenience the logarithm of $\mathrm{L}$ may be maximised. Differentiating $\log \mathrm{L}$ partially with respect to $\theta_{1}, \ldots, \theta_{p}$ and equating to zero we get

$$
\begin{gathered}
\frac{\partial \log \mathrm{L}}{\partial \theta_{i}}=\frac{\partial \log \mathrm{L}_{1}}{\partial \theta_{i}}+\ldots+\frac{\partial \log \mathrm{L}_{k}}{\partial \theta_{i}}=0 \\
i=\mathrm{I}, 2, \ldots, p
\end{gathered}
$$

as the equations giving the desired estimates.

The above equations are usually non-linear and hence the direct evaluation of the estimates is difficult. A general method is to start with trial solutions and derive linear equations giving small corrections to the trial values. The process may have to be repeated until the corrections become negligible. If $d \theta_{1}, \ldots, d \theta_{p}$ are additive correc- 
tions to trial values $\theta_{1}^{0} \ldots, \theta_{p}^{0}$ one derives the equations giving the corrections as

$$
\begin{gathered}
\frac{-\partial^{2} \log \mathrm{L}}{\partial \theta_{i} \partial \theta_{1}} d \theta_{1}-\frac{\partial^{2} \log \mathrm{L}}{\partial \theta_{i} \partial \theta_{2}} d \theta_{2}-\ldots=\frac{\partial \log \mathrm{L}}{\partial \theta_{i}} \\
i=\mathrm{I}, 2, \ldots, p
\end{gathered}
$$

where all the derivatives are calculated at the trial values. If the sample is large we may replace $-\partial^{2} \log \mathrm{L} / \partial \theta_{i} \partial \theta_{j}$ by its mean value which is the same as $\mathrm{I}_{i j}$ the mean value of $\left(\frac{\partial \log \mathrm{L}}{\partial \theta_{i}} \cdot \frac{\partial \log \mathrm{L}}{\partial \theta_{j}}\right)$ at the trial values. The matrix $\mathrm{I}=\left(\mathrm{I}_{i j}\right)$ is called the information matrix for the whole body of data at the assumed values.

The quantity $\partial \log \mathrm{L} / \partial \theta_{i}$ is defined as the $i$-th efficient score and since

$$
\frac{\partial \log \mathrm{L}}{\partial \theta_{i}}=\frac{\partial \log \mathrm{L}_{1}}{\partial \theta_{i}}+\ldots+\frac{\partial \log \mathrm{L}_{k}}{\partial \theta_{i}}
$$

it follows that the efficient scores for the whole data are the sums of the corresponding scores for the several sets of data. Similarly

$$
\mathrm{I}_{i j}=\mathrm{I}_{i j}^{(1)}+\ldots+\mathrm{I}_{i j}^{(k)}
$$

where $\mathrm{I}_{i j}^{(r)}$ is an element of the information matrix for the $r$-th part of the data.

Thus the problem of estimation reduces to replacing each part of the data by the efficient scores and information matrix at some trial values and finally obtaining the corresponding quantities for the whole data by simple addition. These supply linear equations in small additive corrections to the trial values.

It has been demonstrated in section 6 that the method of scoring offers a quickly converging process and hence is extremely useful in practice.

The scores for various parts of the data calculated at the best estimates are directly useful in tests of homogeneity as explained in sections 4 and 5 and illustrated in section 6 . The theoretical aspects of such tests are fully discussed by the author in (Rao, I948).

\section{Notations.-}

$y_{1}, y_{2}, y_{3}$ represent the recombination fractions in the segments connecting the second and third, third and first and first and second loci respectively.

$\dot{y}_{1}, \dot{y}_{2}, \dot{y}_{3}$ are the maximum likelihood estimates when they are treated as free parameters.

$\ddot{y}_{1}, \ddot{y}_{2}, \ddot{y}_{3}$ are the corresponding estimates when Kosambi's formula connecting $y_{1}, y_{2}, y_{3}$ is used. 
$\phi_{1}^{(k)}, \phi_{2}^{(k)}, \phi_{3}^{(k)}$ are the efficient scores for $y_{1}, y_{2}, y_{3}$ treated as free parameters from the $k$-th part of the data. When the data is arranged as frequencies in certain classes, it is known that

$$
\phi_{r}^{(k)}=\mathrm{S}\left(\frac{n}{\pi} \frac{\partial \pi}{\partial y_{r}}\right)
$$

where $\mathrm{S}$ stands for summation, $n$ for the frequency of a class and $\pi$ for the corresponding probability. The total scores for the whole body of data are represented by $\Phi_{1}, \Phi_{2}, \Phi_{3}$.

$\psi_{1}, \psi_{3}$ represent the scores for $y_{1}$ and $y_{3}$ when Kosambi's formula is used.

$\left(i_{r s}^{(k)}\right)$ and $\left(\mathrm{I}_{r s}^{(k)}\right)$ represent the information matrix per single observation and for the whole sample in the $k$-th part of the data. If $n_{k}$ is the sample size then the relation $\left(\mathrm{I}_{r 8}^{(k)}\right)=n_{k}\left(i_{r 8}^{(k)}\right)$ is identically true. It is known that,

$$
i_{r s}^{(k)}=\mathrm{S}\left(\frac{\mathrm{I}}{\pi} \frac{\partial \pi}{\partial y_{r}}\right)_{-} \frac{\partial \pi}{\partial y_{s}}=\mathrm{S} \frac{\partial \pi}{\partial y_{r}}\left(\frac{\mathrm{I}}{\pi} \frac{\partial \pi}{\partial y_{s}}\right)
$$

$\left(\mathrm{T}_{r s}\right)$ or simply denoted by $\mathrm{T}$ stands for the total information $\operatorname{matrix}\left(\mathrm{T}_{r s}\right)=\mathrm{S}\left(\mathrm{I}_{r s}^{(k)}\right)$.

The inverse of $T$ is denoted by $T^{-1}$. The method of finding this is to solve the equations

$$
\begin{aligned}
& p \mathrm{~T}_{11}+q \mathrm{~T}_{12}+r \mathrm{~T}_{13}=\mathrm{I}, \mathrm{o}, \mathrm{o} \\
& p \mathrm{~T}_{12}+q \mathrm{~T}_{22}+r \mathrm{~T}_{23}=\mathrm{o}, \mathrm{I}, \mathrm{o} \\
& p \mathrm{~T}_{13}+q \mathrm{~T}_{23}+r \mathrm{~T}_{33}=\mathrm{o}, \mathrm{o}, \mathrm{I}
\end{aligned}
$$

and take the three sets of equations as the three rows of $\mathrm{T}^{-1}$. $\mathbf{j}$ and $\mathbf{J}$ stand for information matrices per single observation and for the whole body of data when Kosambi's formula is used. All the above quantities calculated at $\dot{y}_{1}, \dot{y}_{2}, \dot{y}_{3}$ and $\ddot{y}_{1}, \ddot{y}_{2}, \ddot{y}_{3}$ are represented with a single dot ànd two dots above respectively.

\section{SCORING OF DATA FROM BACK CROSSES AND INTERCROSSES}

The eight different gametes due to a triply heterozygous parent can be classified into four complementary pairs, the members of each pair having equal chance of being transmitted to an offspring. Let the recombination fractions for the segments connecting the first and second, second and third and third and first loci be represented by $y_{3}, y_{1}$ and $y_{2}$. Also let $\mathrm{S}_{0}, \mathrm{~S}_{1}, \mathrm{~S}_{2}$ and $\mathrm{S}_{3}$ represent respectively the frequencies of gametes involving all three recessive genes, only the first, only the second and only the third recessive gene. The S's and $y$ 's are interrelated in the four types of heterozygotes as given in table I. 
TABLE I

$S$ functions for the four types of triple heterozygotes

Function of $y$ 's

Gametic frequencies for heterozygotes

\begin{tabular}{|c|c|c|c|c|}
\hline & $\frac{\mathrm{ABC}}{\mathrm{abc}}$ & $\frac{\mathrm{AbC}}{\mathrm{aBc}}$ & $\frac{\mathrm{ABc}}{\mathrm{abC}}$ & $\frac{\mathrm{aBC}}{\mathrm{Abc}}$ \\
\hline $\begin{array}{l}\frac{1}{4}\left(2-y_{3}-y_{1}-y_{2}\right) \\
\frac{1}{4}\left(y_{2}+y_{1}-y_{3}\right) \\
\frac{1}{4}\left(y_{3}+y_{1}-y_{2}\right) \\
\frac{1}{4}\left(y_{3}+y_{2}-y_{1}\right)\end{array}$ & $\begin{array}{l}\mathrm{S}_{0} \\
\mathrm{~S}_{3} \\
\mathrm{~S}_{2} \\
\mathrm{~S}_{1}\end{array}$ & $\begin{array}{l}\mathrm{S}_{2} \\
\mathrm{~S}_{1} \\
\mathrm{~S}_{0} \\
\mathrm{~S}_{3}\end{array}$ & $\begin{array}{l}\mathrm{S}_{3} \\
\mathrm{~S}_{0} \\
\mathrm{~S}_{1} \\
\mathrm{~S}_{2}\end{array}$ & $\begin{array}{l}\mathrm{S}_{1} \\
\mathrm{~S}_{2} \\
\mathrm{~S}_{3} \\
\mathrm{~S}_{0}\end{array}$ \\
\hline
\end{tabular}

For a given set of recombination fractions one can, by using the above table, calculate the S-functions for any type of heterozygote and use them for further calculations as the probabilities, scores and information matrix, in any particular situation directly depend on them.

\section{(a) Triple back cross}

The probabilities of the eight phenotypical classes and the appropriate scores, expressed in terms of S-functions, in the case of a back cross of a triple heterozygote with a triple recessive are given in table 2.

TABLE 2

Probabilities and scores in the case of a triple back cross

\begin{tabular}{|c|c|c|c|c|c|}
\hline Phenotype & Probability & $y_{1}$ & $\begin{array}{c}\text { Scores for } \\
y_{2}\end{array}$ & $y_{3}$ & $\begin{array}{l}\text { Observed } \\
\text { frequency }\end{array}$ \\
\hline $\mathrm{ABC}$ & $\mathrm{S}_{0}$ & $l_{0} / 4 \mathrm{~S}_{0}$ & $m_{0} / 4 \mathrm{~S}_{0}$ & $n_{0} / 4 \mathrm{~S}_{0}$ & $n_{111}$ \\
\hline $\mathrm{AbC}$ & $\mathrm{S}_{2}$ & $l_{2} / 4 \mathrm{~S}_{2}$ & $m_{2} / 4 \mathrm{~S}_{2}$ & $n_{2} / 4 \mathrm{~S}_{2}$ & $n_{101}$ \\
\hline $\mathrm{ABc}$ & $\mathrm{S}_{3}$ & $l_{3} / 4 \mathrm{~S}_{3}$ & $m_{3} / 4 \mathrm{~S}_{3}$ & $n_{3} / 4 \mathrm{~S}_{3}$ & $n_{110}$ \\
\hline $\mathrm{Abc}$ & $\mathrm{S}_{1}^{0}$ & $l_{1} / 4 \mathrm{~S}_{1}$ & $m_{1} / 4 \mathrm{~S}_{1}$ & $n_{1} / 4 \mathrm{~S}_{1}$ & $n_{100}$ \\
\hline $\mathrm{aBC}$ & $S_{1}$ & $l_{1} / 4 \mathrm{~S}_{1}$ & $m_{1} / 4 \mathrm{~S}_{1}$ & $n_{1} / 4 \mathrm{~S}_{1}$ & $n_{011}$ \\
\hline $\mathrm{abC}$ & $\mathrm{S}_{3}$ & $l_{3} / 4 \mathrm{~S}_{3}$ & $m_{3} / 4 \mathrm{~S}_{3}$ & $n_{3} / 4 \mathrm{~S}_{3}$ & $n_{001}$ \\
\hline $\mathrm{aBc}$ & $\mathrm{S}_{2}$ & $l_{2} / 4 \mathrm{~S}_{2}$ & $m_{2} / 4 \mathrm{~S}_{2}$ & $n_{2} / 4 \mathrm{~S}_{2}$ & $n_{010}$ \\
\hline$a b c$ & $\mathrm{~S}_{0}$ & $l_{0} / 4 \mathrm{~S}_{0}$ & $m_{0} / 4 \mathrm{~S}_{0}$ & $n_{0} / 4 \mathrm{~S}_{0}$ & $n_{000}$ \\
\hline
\end{tabular}

The $l$ 's, $m$ 's and $n$ 's of the above table are four times the derivatives of probabilities with respect to $y_{1}, y_{2}$ and $y_{3}$ respectively and their values are determined by the following rule. The value of the derivative of the probability for a phenotype with respect to $y_{i}$ is $-\frac{1}{4}$ or $\frac{1}{4}$ according as the letters other than the $i$-th in the representation of the phenotype is an old or a new combination, the old combinations being determined by the representation of the triple heterozygote. Thus in deciding the values of $l_{0}, l_{2}, l_{3}$ and $l_{1}$, one need only find which of the combinations $\mathrm{BC}, \mathrm{bC}, \mathrm{Bc}, \mathrm{bc}$, are old and which are new and take the $l$ 's corresponding to old combinations as $-\mathrm{I}$ and the rest as $+\mathrm{r}$. The values of these coefficients for the various types of heterozygotes are given in table 3 for ready use. 
The efficient scores at any given values of $y_{1}, y_{2}$ and $y_{3}$ are obtained by summing the products of observed frequencies and scores. The

\section{TABLE 3}

Values of the l's, m's and n's of table 2

\begin{tabular}{cl} 
Heterozygote & \multicolumn{1}{c}{ Values of the coefficients } \\
$\mathrm{ABC} / \mathrm{abc}$ & $l_{0}=l_{1}=m_{0}=m_{2}=n_{0}=n_{3}=-\mathrm{I}$ \\
& $l_{2}=l_{3}=m_{1}=m_{3}=n_{1}=n_{2}=\mathrm{I}$ \\
$\mathrm{AbC} / \mathrm{aBc}$ & $l_{2}=l_{3}=m_{0}=m_{2}=n_{1}=n_{2}=-\mathrm{I}$ \\
& $l_{0}=l_{1}=m_{1}=m_{3}=n_{0}=n_{3}=\mathrm{I}$ \\
$\mathrm{Abc} / \mathrm{aBC}$ & $l_{0}=l_{1}=m_{1}=m_{3}=n_{1}=n_{2}=-\mathrm{I}$ \\
& $l_{2}=l_{3}=m_{0}=m_{2}=n_{0}=n_{3}=\mathrm{I}$ \\
$\mathrm{ABc} / \mathrm{abC}$ & $l_{2}=l_{3}=m_{1}=m_{3}=n_{0}=n_{3}=-\mathrm{I}$ \\
& $l_{0}=l_{1}=m_{0}=m_{2}=n_{1}=n_{2}=\mathrm{I}$
\end{tabular}

elements of the information matrix $\left(i_{r s}\right)$ per single observation for given values of $y_{1}, y_{2}, y_{3}$ are the same for all types of back crosses. They can be simply calculated by using the formulæ

$$
\begin{aligned}
& i_{r r}=\frac{1}{2}\left(\lambda_{0}+\lambda_{1}+\lambda_{2}+\lambda_{3}\right) \quad r=\mathrm{I}, 2,3 \\
& i_{12}=\frac{1}{2}\left(\lambda_{0}-\lambda_{1}-\lambda_{2}+\lambda_{3}\right) \\
& i_{23}=\frac{1}{2}\left(\lambda_{0}+\lambda_{1}-\lambda_{2}-\lambda_{3}\right) \\
& i_{13}=\frac{1}{2}\left(\lambda_{0}-\lambda_{1}+\lambda_{2}-\lambda_{3}\right)
\end{aligned}
$$

where $\lambda_{0}=\mathrm{r} /\left(2-y_{1}-y_{2}-y_{3}\right), \lambda_{1}=\mathrm{r} /\left(y_{2}+y_{3}-y_{1}\right), \lambda_{2}=\mathrm{r} /\left(y_{1}+y_{3}-y_{2}\right)$ and $\lambda_{3}=\mathrm{r} /\left(y_{1}+y_{2}-y_{3}\right)$. It may be noted that the $\lambda^{\prime}$ s are fixed functions of the recombination fractions and any S-function assumes one of the values of $\lambda$ for a given heterozygote. These have been introduced merely to simplify computations.

If the whole data consist only of results from back crosses the maximum likelihood estimates can be obtained without the evaluation of the efficient scores. It is easily seen that for such data the equations giving the best estimates are

$$
\begin{aligned}
& \text { (o) } \lambda_{0}+(\mathrm{I}) \lambda_{1}-(\mathrm{I} 2) \lambda_{2}-(2) \lambda_{3}=0 \\
& \text { (o) } \lambda_{0}-\text { (1) } \lambda_{1}+(\mathrm{I} 2) \lambda_{2}-(2) \lambda_{3}=0 \\
& \text { (o) } \lambda_{0}-(\mathrm{I}) \lambda_{1}-(\mathrm{I} 2) \lambda_{2}+(2) \lambda_{3}=0
\end{aligned}
$$

where (o), (1), (2) and (12) are the totals of observed frequencies from all sets of data corresponding to no cross overs, cross overs in the first segment only, cross overs in the second segment only and double cross overs respectively.

These equations yield the solutions

$$
\text { (o) } \lambda_{0}=(\text { I }) \lambda_{1}=(\text { I } 2) \lambda_{2}=(2) \lambda_{3}
$$


or, writing in terms of the $y$ 's, they become

$$
\begin{aligned}
& \frac{(\mathrm{o})}{2-y_{1}-y_{2}-y_{3}}=\frac{(\mathrm{I})}{y_{2}+y_{3}-y_{1}}=\frac{(\mathrm{I} 2)}{y_{1}+y_{3}-y_{2}}=\frac{(2)}{y_{1}+y_{2}-y_{3}}= \\
& \frac{(1)+(\mathrm{I} 2)}{2 y_{3}}=\frac{(2)+(12)}{2 y_{1}}=\frac{(1)+(2)}{2 y_{2}}=\frac{(\mathrm{o})+(\mathrm{I})+(2)+(12)}{2}=\frac{\mathrm{N}}{2} .
\end{aligned}
$$

Hence $\dot{y}_{1}=\frac{(2)+(12)}{\mathrm{N}}, \quad \dot{y}_{2}=\frac{(1)+(2)}{\mathrm{N}}, \quad \dot{y}_{3}=\frac{(\mathrm{I})+(\mathrm{I} 2)}{\mathrm{N}}$.

The above estimates for $y_{1}, y_{2}$ and $y_{3}$ are the same as those obtained by considering the data as classified according two factors each time. Thus $\dot{y}_{1}$ could be obtained by considering only the second and third factors and ignoring the classification due to the first factor. Thus a complete classification with respect to three factors in the case of back crosses does not supply additional information so far as the problem of estimation of recombination fractions in various segments is concerned. This is true with more than three factors also.

The variances and covariances of the above estimates are

$$
\mathrm{V}\left(\dot{y}_{1}\right)=\frac{y_{1}\left(\mathrm{I}-y_{1}\right)}{\mathrm{N}}, \quad \mathrm{V}\left(\dot{y}_{2}\right)=\frac{y_{2}\left(\mathrm{r}-y_{2}\right)}{\mathrm{N}}, \quad \mathrm{V}\left(\dot{y}_{3}\right)=\frac{y_{3}\left(\mathrm{I}-y_{3}\right)}{\mathrm{N}}
$$

Cov. $\left(\dot{y}_{1} \dot{y}_{2}\right)=\frac{y_{1}+y_{2}-y_{3}-2 y_{1} y_{2}}{2 \mathrm{~N}}, \quad$ Cov. $\left(\dot{y}_{2} \dot{y}_{3}\right)=\frac{y_{2}+y_{3}-y_{1}-2 y_{2} y_{3}}{2 \mathrm{~N}}$

Cov. $\left(\dot{y}_{1} \dot{y}_{3}\right)=\frac{y_{1}+y_{3}-y_{2}-2 y_{1} y_{3}}{2 \mathrm{~N}}$

(b) Intercross of a triple heterozygote

The frequencies and their derivatives in terms of S-functions for any type of intercross are given in table 4 .

TABLE 4

Frequencies and their derivatives for an intercross

Phenotype

Probability

$$
\pi \quad \frac{\partial \pi}{\partial y_{1}}
$$

Derivatives

$\begin{array}{lrlll}\mathrm{ABC} & \frac{1}{4}+\mathrm{S}_{0}+\mathrm{S}_{1}^{2}+\mathrm{S}_{2}^{2}+\mathrm{S}_{3}^{2} & l_{0}\left(\mathrm{~S}_{0}+2 \mathrm{~S}_{1}\right) & m_{0}\left(\mathrm{~S}_{0}+2 \mathrm{~S}_{2}\right) & n_{0}\left(\mathrm{~S}_{0}+2 \mathrm{~S}_{3}\right) \\ \mathrm{AbC} & \mathrm{S}_{2}-\mathrm{S}_{2}^{2}+2 \mathrm{~S}_{1} \mathrm{~S}_{3} & l_{2}\left(\mathrm{~S}_{0}+2 \mathrm{~S}_{1}\right) & m_{2} \mathrm{~S}_{0} & n_{2}\left(\mathrm{~S}_{0}+2 \mathrm{~S}_{3}\right) \\ \mathrm{ABc} & \mathrm{S}_{3}-\mathrm{S}_{3}^{2}+2 \mathrm{~S}_{1} \mathrm{~S}_{2} & l_{3}\left(\mathrm{~S}_{0}+2 \mathrm{~S}_{1}\right) & m_{3}\left(\mathrm{~S}_{0}+2 \mathrm{~S}_{2}\right) & n_{3} \mathrm{~S}_{0} \\ \mathrm{Abc} & \mathrm{S}_{1}^{2}+2 \mathrm{~S}_{1} \mathrm{~S}_{0} & l_{1}\left(\mathrm{~S}_{0}+2 \mathrm{~S}_{1}\right) & m_{1} \mathrm{~S}_{0} & n_{1} \mathrm{~S}_{0} \\ \mathrm{aBC} & \mathrm{S}_{1}-\mathrm{S}_{1}^{2}+2 \mathrm{~S}_{2} \mathrm{~S}_{3} & l_{1} \mathrm{~S}_{0} & m_{1}\left(\mathrm{~S}_{0}+2 \mathrm{~S}_{2}\right) & n_{1}\left(\mathrm{~S}_{0}+2 \mathrm{~S}_{3}\right) \\ \mathrm{abC} & \mathrm{S}_{3}^{2}+2 \mathrm{~S}_{3} \mathrm{~S}_{0} & l_{3} \mathrm{~S}_{0} & m_{3} \mathrm{~S}_{0} & n_{3}\left(\mathrm{~S}_{0}+2 \mathrm{~S}_{3}\right) \\ \mathrm{aBc} & \mathrm{S}_{2}^{2}+2 \mathrm{~S}_{2} \mathrm{~S}_{0} & l_{2} \mathrm{~S}_{0} & m_{2}\left(\mathrm{~S}_{0}+2 \mathrm{~S}_{2}\right) & n_{2} \mathrm{~S}_{0} \\ \mathrm{abc} & \mathrm{S}_{0}^{2} & l_{0} \mathrm{~S}_{0} & m_{0} \mathrm{~S}_{0} & n_{0} \mathrm{~S}_{0}\end{array}$

The $l$ 's in the column for $\hat{c} \pi / \partial y_{1}$ are $-\frac{1}{2}$ or $\frac{1}{2}$ according as the factors other than the first in the representation of the corresponding 
phenotypes form old or new combinations. These can be readily obtained from table 3 by replacing unity by $\frac{1}{2}$. The appropriate scores, which are used in the calculation of efficient scores, for any class, are calculated by dividing the derivatives by the class probability. The information matrix is evaluated by the formulæ

$$
\begin{aligned}
& \mathrm{I}_{r r}=n \mathrm{~S}\left(\frac{\mathrm{I}}{\pi} \frac{\partial \pi}{\partial y_{r}}\right) \frac{\partial \pi}{\partial y_{r}} \\
& \mathrm{I}_{r k}=n \mathrm{~S}\left(\frac{\mathrm{I}}{\pi} \frac{\partial \pi}{\partial y_{r}}\right) \frac{\partial \pi}{\partial y_{k}}=n \mathrm{~S} \frac{\partial \pi}{\partial y_{r}}\left(\frac{\mathrm{I}}{\pi} \frac{\partial \pi}{\partial y_{k}}\right)
\end{aligned}
$$

where $n$ is the total of observed frequencies. The maximum likelihood estimates, in this case, are obtained by successive approximations as explained in the next section.

\section{MAXIMUM LIKELIHOOD ESTIMATES OF THE RECOMBINATION FRACTIONS TAKEN AS FREE PARAMETERS}

When the data from various sources and different types of crosses giving information on only one or all the three recombination fractions are available, there arise the problems of obtaining the best estimates from the combined data and testing homogeneity of different parts of the data. The numerical computation of the above problems can be arranged as follows. To start with the scores and the information matrix, at the approximate values of $y_{1}, y_{2}, y_{3}$ are calculated for separate portions of the data as shown in table 5 .

\begin{tabular}{|c|c|c|c|c|c|c|c|c|c|}
\hline \multirow{2}{*}{$\begin{array}{l}\text { Effic } \\
\text { Source and type } \\
\text { of cross } \\
\text { I }\end{array}$} & \multicolumn{3}{|c|}{$\begin{array}{l}\text { scores and information } \\
\text { Efficient scores }\end{array}$} & \multicolumn{6}{|c|}{$\begin{array}{l}\text { 2atrix at the approximate values } \\
\text { Information matrix }\end{array}$} \\
\hline & $\phi_{1}^{(1)}$ & $\phi_{2}^{(1)}$ & $\phi_{3}^{(1)}$ & $I_{11}^{(1)}$ & $I_{12}^{(1)}$ & $\mathrm{I}_{13}^{(1)}$ & $\mathrm{I}_{22}^{(1)}$ & $\mathrm{I}_{23}^{(1)}$ & $\mathbf{I}_{33}^{(1)}$ \\
\hline · & $\cdot$ & 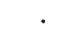 & . & $\cdot$ & 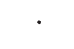 & & . & . & \\
\hline$k$ & $\phi_{1}^{(k)}$ & $\phi_{2}^{(k)}$ & $\phi_{3}^{(k)}$ & $\mathbf{I}_{11}^{\left(k^{\prime}\right)}$ & $\mathrm{I}_{12}^{(k)}$ & $\mathbf{I}_{13}^{\left(k^{k}\right)}$ & $\mathrm{I}_{22}^{(k)}$ & $\mathrm{I}_{23}^{(k)}$ & $\mathbf{I}_{33}^{(k)}$ \\
\hline Total & $\Phi_{1}$ & $\Phi_{2}$ & $\Phi_{3}$ & $\mathrm{~T}_{11}$ & $\mathrm{~T}_{12}$ & $\mathrm{~T}_{13}$ & $\mathrm{~T}_{22}$ & $\mathrm{~T}_{23}$ & $T_{33}$ \\
\hline
\end{tabular}

TABLE 5

In the above table, if any part of the data gives information for only one segment, say the first, then only $\phi_{3}$ and $\mathrm{I}_{33}$ are present and the rest are zero for that part. The methods of scoring in such cases have been fully discussed by Fisher (1946) and Bhat (I947) and also illustrated in this article in section $7(c)$. For scoring parts of the data giving the simultaneous segregation of three factors the expressions derived in section 3 may be used.

Using the totals of table 5 , the three linear equations in $d y_{1}, d y_{2}$ and $d y_{3}$, which are additive corrections to the first approximations of $y_{1}, y_{2}$ and $y_{3}$ respectively, can be written

$$
\begin{array}{llll}
\mathrm{T}_{11} & d y_{1}+\mathrm{T}_{12} & d y_{2}+\mathrm{T}_{13} & d y_{3}=\Phi_{1} \\
\mathrm{~T}_{12} & d y_{1}+\mathrm{T}_{22} & d y_{2}+\mathrm{T}_{23} & d y_{3}=\Phi_{2} \\
\mathrm{~T}_{13} & d y_{1}+\mathrm{T}_{23} & d y_{2}+\mathrm{T}_{33} & d y_{3}=\Phi_{3}
\end{array}
$$


These calculations require to be repeated until the values of $y_{1}$, $y_{2}$ and $y_{3}$ for which the total scores are negligible are obtained.

If the scores and information matrix for the $i$-th part of the data at the estimated values are represented by

$$
\dot{\phi}_{1}^{(i)}, \dot{\phi}_{2}^{(i)}, \dot{\phi}_{3}^{(i)} \text { and }\left(\dot{\mathrm{I}}_{r s}^{(i)}\right)
$$

then using the elements of the matrix $\left(\dot{\mathrm{I}}_{(i)}^{r g}\right)$ reciprocal to $\left(\dot{\mathrm{I}}_{r s}^{(i)}\right)$ a $\chi^{2}$ can be calculated by the formula

$$
\chi_{i}^{2}=\operatorname{SS} \dot{\mathrm{I}}_{(i)}^{r s} \dot{\phi}_{r}^{(i)} \dot{\phi}_{s}^{(i)}
$$

If the $i$-th part of the data concerns only two of the factors, say the first and second, then $\chi_{i}^{2}$ is simply $\left[\dot{\phi}_{3}^{(i)}\right]^{2} / \dot{I}_{33}^{(i)}$. To test for heterogeneity the total

$$
\chi^{2}=\chi_{1}^{2}+\ldots+\chi_{k}^{2}
$$

can be used as $\chi^{2}$ with degrees of freedom

$$
d_{1}+\ldots+d_{k}-3
$$

where $d_{i}=\mathrm{I}$ or 3 according as the $i$-th part of the data relates to the segregation of only two or three factors. A significant $\chi^{2}$ at a chosen probability level indicates that different parts of the data are not homogeneous.

\section{MAXIMUM LIKELIHOOD ESTIMATES SUBJECT TO KOSAMBI'S FORMULA}

The general formula $\tanh 2 x=2 y$ giving the relation between the map distance $x$ and the recombination fraction $y$ is given by Kosambi (1944). In terms of $y_{1}, y_{2}$ and $y_{3}$, the relation becomes

$$
y_{2}=\frac{y_{1}+y_{3}}{\mathrm{I}+4 y_{1} y_{3}}
$$

There are only two parameters $y_{1}$ and $y_{3}$ to be estimated, the third one, $y_{2}$, being obtainable from the above formula. The scores and information matrix for $y_{1}$ and $y_{3}$ can be conveniently calculated from the corresponding expressions for $y_{1}, y_{2}$ and $y_{3}$ taken as free parameters and in any practical problem the data may be scored for $y_{1}, y_{2}$ and $y_{3}$ taken as free parameters and then the appropriate scores for $y_{1}$ and $y_{3}$, when Kosambi's formula is applicable, can be deduced. If $\psi_{1}$ and $\psi_{3}$ represent the total scores for $y_{1}$ and $y_{3}$ and $\mathrm{J}_{11}, \mathrm{~J}_{13}, \mathrm{~J}_{33}$, the elements of the information matrix then the formulæ connecting them with the totals of table 5 are

$$
\begin{gathered}
\psi_{1}=\Phi_{1}+\mu_{1} \Phi_{2} \quad, \quad \mu_{1}=\left(\mathrm{I}-4 y_{3}^{2}\right) /\left(\mathrm{I}+4 y_{1} y_{3}\right)^{2} \\
\psi_{3}=\Phi_{3}+\mu_{3} \Phi_{2} \quad, \quad \mu_{3}=\left(\mathrm{I}-4 y_{3}^{2}\right) /\left(\mathrm{I}+4 y_{1} y_{3}\right)^{2} \\
\mathrm{~J}_{11}=\mathrm{T}_{11}+2 \mu_{1} \mathrm{~T}_{12}+\mu_{1}^{2} \mathrm{~T}_{22} \\
\mathrm{~J}_{13}=\mathrm{T}_{13}+\mu_{1} \mathrm{~T}_{23}+\mu_{3} \mathrm{~T}_{12}+\mu_{1} \mu_{3} \mathrm{~T}_{22} \\
\mathrm{~J}_{33}=\mathrm{T}_{33}+2 \mu_{3} \mathrm{~T}_{23}+\mu_{3}^{2} \mathrm{~T}_{22}
\end{gathered}
$$


The additive corrections $d y_{1}$ and $d y_{3}$ to approximate values of $y_{1}$ and $y_{3}$ are obtained from the equations

$$
\begin{array}{lll}
\mathrm{J}_{11} & d y_{1}+\mathrm{J}_{13} & d y_{3}=\psi_{1} \\
\mathrm{~J}_{13} & d y_{1}+\mathrm{J}_{33} & d y_{3}=\psi_{3}
\end{array}
$$

If $\ddot{\Phi}_{2}$ is the total score for $y_{2}$ and $\left(\ddot{\mathrm{T}}^{r}\right)$ is the inverse of the total information matrix calculated as in table 5 for $y_{1}, y_{2}, y_{3}$ at the estimated values $\ddot{y}_{1}, \ddot{y}_{2}, \ddot{y}_{3}$, subject to Kosambi's formula, then

$$
\chi^{2}=\ddot{\Phi}_{2}^{2}\left(\ddot{\mathrm{T}}^{22}+\mu_{1}^{2} \ddot{\mathrm{T}}^{11}+\mu_{3}^{2} \ddot{\mathrm{T}}^{33}+2 \mu_{1} \mu_{3} \ddot{\mathrm{T}}^{13}-2 \mu_{1} \ddot{\mathrm{T}}^{12}-2 \mu_{3} \ddot{\mathrm{T}}^{23}\right)
$$

can be used as $\chi^{2}$ with I d.f. to test the agreement with Kosambi's formula. The estimates, obtained subject to Kosambi's formula, are valid only when the above test does not show significance.

\section{AN ILLUSTRATIVE EXAMPLE}

The methods developed in sections 3,4 and 5 are applied to the linkage data for Primula sinensis reproduced from a paper by de Winton and Haldane (I935).

The data chosen consist of two types of back crosses so that exact values of maximum likelihood estimates could be obtained. The method of scoring can also be applied, starting with some trial values, and the first approximations which, in this case, must be identical with the exact values may be obtained.

The trial values chosen are $y_{1}=\cdot 35, y_{2}=.39, y_{3}=.07$ so that,

$$
\begin{array}{llr}
\lambda_{0}=\mathrm{I} /\left(2-y_{1}-y_{2}-y_{3}\right) & =\mathrm{I} / \mathrm{I} \cdot \mathrm{I} 9= & .840336 \\
\lambda_{1}=\mathrm{I} /\left(y_{2}+y_{3}-y_{1}\right) & =\mathrm{I} / \cdot \mathrm{I} \mathbf{I}=9.090909 \\
\lambda_{2}=\mathrm{I} /\left(y_{1}+y_{3}-y_{2}\right) & =\mathrm{I} / \cdot 03=33.333333 \\
\lambda_{3}=\mathrm{I} /\left(y_{1}+y_{2}-y_{3}\right) & =\mathrm{I} / \cdot 67=\mathrm{r} \cdot 492537
\end{array}
$$

\begin{tabular}{|c|c|c|c|c|c|c|}
\hline \multirow{3}{*}{$\begin{array}{c}\text { Phenotype } \\
\text { SBL }\end{array}$} & \multicolumn{2}{|r|}{ Set I } & \multicolumn{2}{|r|}{$\frac{\mathrm{SBL}}{\mathrm{sbl}}$} & & \multirow[b]{2}{*}{ Multiplier } \\
\hline & $\begin{array}{l}\text { Observed } \\
\text { frequency }\end{array}$ & $\begin{array}{l}\text { Type of } \\
\text { cross over }\end{array}$ & $y_{1}$ & $\begin{array}{c}\text { Scores for } \\
y_{2}\end{array}$ & $y_{3}$ & \\
\hline & 457 & (o) & - & - & - & $.840336=\mathrm{I} / 4 \mathrm{~S}_{0}$ \\
\hline $\mathrm{SbL}$ & I I & (12) & + & - & + & $33 \cdot 333333=\mathrm{I} / 4 \mathrm{~S}_{2}$ \\
\hline $\mathrm{SBl}$ & 256 & (2) & + & + & - & $\mathrm{I} \cdot 492537=\mathrm{I} / 4 \mathrm{~S}_{3}$ \\
\hline $\mathrm{Sbl}$ & $3^{8}$ & (I) & - & + & + & $9 \cdot 090909=\mathrm{i} / 4 \mathrm{~S}_{1}^{\circ}$ \\
\hline sBL & 45 & (I) & 一 & + & + & $9^{\cdot} \circ g^{\circ} \circ \operatorname{cog}=\mathrm{I} / 4 \mathrm{~S}_{1}$ \\
\hline $\mathrm{sbL}$ & 284 & (2) & + & + & - & $\mathrm{I} \cdot 492537=\mathrm{I} / 4 \mathrm{~S}_{3}$ \\
\hline sBl & 20 & (12) & + & - & + & $33 \cdot 333333=\mathrm{I} / 4 \mathrm{~S}_{2}$ \\
\hline $\mathrm{sbl}$ & 469 & (o) & - & - & - & $.840336=\mathrm{I} / 4 \mathrm{~S}_{0}$ \\
\hline & 80 & & & & & \\
\hline
\end{tabular}

The $\lambda$ 's are one-fourth of the reciprocals of the S-functions so that for any back cross the scores given in table 2 are obtained by multiplying the $\lambda$ 's with the appropriate values of the $l$ 's, $m$ 's and $n$ 's given in table 3 .

\section{TABLE 6}

Linkage data of SBL in Primula sinensis (오 side) 


\begin{tabular}{|c|c|c|c|c|c|c|}
\hline \multirow[b]{2}{*}{ Phenotype } & \multirow{2}{*}{\multicolumn{2}{|c|}{$\begin{array}{l}\text { Set II } \\
\text { Type of } \\
\text { cross over }\end{array}$}} & $\frac{\mathrm{sbl}}{\mathrm{sbl}}$ & \multicolumn{2}{|c|}{$\frac{\mathrm{SBl}}{\mathrm{sbL}}$} & \multirow[b]{2}{*}{ Multiplier } \\
\hline & & & $y_{1}$ & $\begin{array}{c}\text { cores } \\
y_{2}\end{array}$ & $y_{8}$ & \\
\hline SBL & $2 \mathrm{I}$ & $(2)$ & + & + & - & $\mathrm{I} \cdot 49^{2} 537=\mathrm{I} / 4 \mathrm{~S}_{0}$ \\
\hline $\mathrm{SbL}$ & 3 & (I) & - & + & + & $9 \cdot 090909=1 / 4 S_{2}$ \\
\hline $\mathrm{SBl}$ & 50 & (o) & - & 一 & 一 & $\cdot 840336=1 / 4 S_{3}$ \\
\hline Sbl & I & (12) & + & 一 & + & $33 \cdot 333333=1 / 4 \mathrm{~S}_{1}$ \\
\hline sBL & 1 & (12) & + & - & + & $33 \cdot 333333=\mathrm{I} / 4 \mathrm{~S}_{3}$ \\
\hline $\mathrm{sbL}$ & 57 & (o) & - & - & - & $\cdot 840336=1 / 4 \mathrm{~S}_{3}$ \\
\hline $\mathrm{sBl}$ & 4 & (I) & - & + & + & $9 \cdot 090909=1 / 4 \mathrm{~S}_{2}$ \\
\hline $\mathrm{sbl}$ & 26 & (2) & + & + & - & $\mathrm{I} \cdot 492537=\mathrm{I} / 4 \mathrm{~S}_{0}$ \\
\hline
\end{tabular}

The exact values from the combined data are given by

$$
\begin{aligned}
& \dot{y}_{1}=\frac{(2)+(\mathrm{I} 2)}{\mathrm{N}}=\frac{(256+284+\mathrm{II}+20)+(2 \mathrm{I}+\mathrm{I}+\mathrm{I}+26)}{\mathrm{I} 580+\mathrm{I} 63} \\
& =\frac{620}{\mathrm{I} 743}=\cdot 355709 \\
& \dot{y}_{2}=\frac{(\mathrm{I})+(2)}{\mathrm{N}}=\frac{677}{\mathrm{I} 743}=\cdot 3884 \mathrm{II} \\
& \dot{y}_{3}=\frac{(\mathrm{I})+(\mathrm{I} 2)}{\mathrm{N}}=\frac{123}{\mathrm{I} 743}=.070568
\end{aligned}
$$

The efficient scores at the trial values.

$$
\begin{array}{lrrr}
\text { Set I } & 306 \cdot 606720 & -250 \cdot 969032 & 203.757654 \\
\text { Set II } & -16.736410 & -22 \cdot 797016 & -29 \cdot 762162 \\
\quad \text { Total }= & 289.8703 \text { I } & -273.766048 & \text { I } 73.995492
\end{array}
$$

If the data contained some parts giving only two factors segregations then the scores arising from them have to be added to the above values. Such data can supply only one of the efficient scores, the others being considered as zero. In such cases the appropriate scores for intercrosses are given on p. 6I and for back crosses on p. 58 in Mather's book, The Measurement of Linkage in Heredity (first edition).

The information matrix per single observation is the same for both the above sets at the trial values.

$$
\begin{aligned}
& i_{11}=i_{22}= i_{33}=\frac{1}{2}\left(\lambda_{0}+\lambda_{1}+\lambda_{2}+\lambda_{3}\right)=22 \cdot 378557 \\
& i_{12}=\frac{1}{2}\left(\lambda_{0}-\lambda_{1}-\lambda_{2}+\lambda_{3}\right)=-20 \cdot 045685 \\
& i_{23}=\frac{1}{2}\left(\lambda_{0}+\lambda_{1}-\lambda_{2}-\lambda_{3}\right)=- \text { I } 2 \cdot 4473 \text { I } \\
& i_{13}=\frac{1}{2}\left(\lambda_{0}-\lambda_{1}+\lambda_{2}-\lambda_{3}\right)= \text { I I.795 I I I }
\end{aligned}
$$

The total information matrix $T$ is

$$
T=\left(I_{5} 80+163\right)\left(\begin{array}{rrr}
22 \cdot 378557 & -20 \cdot 045685 & \text { II } \cdot 795 \text { I I I } \\
-20 \cdot 045685 & 22 \cdot 378557 & -12 \cdot 4473 \text { I } 2 \\
\text { I I.795 II I } & -12 \cdot 4473 \text { I } 2 & 22 \cdot 378557
\end{array}\right)
$$


and its inverse is

$$
\mathbf{T}^{-1}=\frac{\mathrm{I}}{\mathrm{I} 743}\left(\begin{array}{rlr}
\cdot 227500 & \cdot 198500 & -\cdot 009500 \\
\cdot 198500 & \cdot 237900 & \cdot 027700 \\
-\cdot 009500 & \cdot 027700 & \cdot 065100
\end{array}\right)
$$

The corrections to trial values are given by

$$
\begin{aligned}
& d y_{i}=\Phi_{1} T^{i 1}+\Phi_{2} T^{i 2}+\Phi_{3} T^{i 3} \\
& d y_{1}=\frac{\mathrm{r}}{\mathrm{I} 743}[\cdot 2275(289 \cdot 8703 \mathrm{Io})+\cdot 1985(-273 \cdot 766048) \\
& \text {-.0095( ( } 73 \cdot 995492)]=\quad \cdot 00570953 \\
& d y_{2}=\frac{I}{1743}[\cdot 1985( \\
& )+\cdot 2379(\quad) \\
& +\cdot 0277()]=-\cdot 00158922 \\
& d y_{3}=\frac{1}{1743}[-\cdot 0095( \\
& )+0277( \\
& +\cdot 065 \mathrm{I}( \\
& )]=0005^{6} 7990
\end{aligned}
$$

The first approximations to trial values $\cdot 35, \cdot 39, \cdot 07$, are

$$
\cdot 355709, \cdot 3884 \text { I I, } 070568
$$

agreeing accurately up to the number of significant figures maintained in the calculations with the exact estimates

$$
\cdot 355709, \cdot 3884 \text { I I, } 070568
$$

obtained earlier. This is not generally true but if the trial values of the cross over percentages can be correctly guessed to the nearest whole numbers the first approximations obtained by the method of scoring are expected to be sufficiently accurate.

The test of the hypothesis that the two sets of data arise from identical values of recombination fractions involves the evaluation of the scores and information matrices at the estimated values. The new scores can, however, be obtained approximately by certain adjustments of the scores at the trial values. The change in the matrix inverse to the information matrix is negligible so that no adjustment is necessary. But in cases where approximations differ considerably from the trial values it is necessary to calculate the scores and information matrix directly.

The adjusted scores for the first set are

$$
\begin{aligned}
& \dot{\phi}_{1}^{(1)}=\phi_{1}^{(1)}-n_{1}\left(d y_{1} i_{11}+d y_{2} i_{12}+d y_{3} i_{13}\right) \\
& =289 \cdot 8703 \text { I0-I } 580[22 \cdot 378557(\cdot 005709)-20 \cdot 045685(\text { - oo I } 589) \\
& +11 \cdot 795 \text { III }(\cdot 000568)] \\
& =289 \cdot 870310-262 \cdot 771854=27 \cdot 09845^{6} \\
& \dot{\phi}_{2}^{(1)}=-273 \cdot 766048-1580[-20 \cdot 045685(\quad)+22 \cdot 378557() \\
& =-273 \cdot 766048+248 \cdot 17 \text { I } 256=-25 \cdot 59479^{2} \\
& -12 \cdot 447312( \\
& \dot{\phi}_{3}^{(1)}=173 \cdot 995492-1580 \text { [I I.795 III ( ) - I } 2 \cdot 4473 \text { I } 2( \\
& =\mathrm{r} 73 \cdot 995492-\mathrm{r} 57 \cdot 728377=\mathrm{r} 6 \cdot 267 \text { I } 5 \\
& \begin{array}{r}
-12 \cdot 447312( \\
+22 \cdot 378557(
\end{array}
\end{aligned}
$$


The value of $\chi_{1}^{2}$ is,

$$
\begin{aligned}
& =\frac{\mathrm{I}}{n_{1}} \mathrm{SS} i^{r s} \dot{\phi}_{r}^{(1)} \dot{\phi}_{8}^{(1)} \\
& =\frac{\mathrm{I}}{\mathrm{r} 58 \mathrm{o}}\left[\cdot 2275(27 \cdot 0984)^{2}+\cdot 2379(25 \cdot 5948)^{2}+\cdot 065 \mathrm{I}(\mathrm{r} 6 \cdot 267 \mathrm{I})^{2}\right. \\
& +2(\cdot 1985)(27 \cdot 0984)(-25 \cdot 5948) \\
& +2(-.0095)(\quad)(16.2671) \\
& +2(\cdot 0277)(-25 \cdot 5948)(\quad)] \\
& =\frac{33 \cdot 3^{807}}{\mathrm{r} 5^{80}}=\cdot 02 \mathrm{II} 3 \text {. }
\end{aligned}
$$

Similarly

$$
\begin{aligned}
& \dot{\phi}_{1}^{(2)}=-27 \cdot 098456, \quad \dot{\phi}_{2}^{(2)}=25.594792, \quad \dot{\phi}_{3}^{(2)}=-16 \cdot 267 \text { I I } 5 \text { and } \\
& \chi_{2}^{2}=\frac{33}{\mathrm{r} 63}=\cdot 2048 .
\end{aligned}
$$

The total $\chi^{2}=\chi_{1}^{2}+\chi_{2}^{2}=\cdot 02 \mathrm{II}+\cdot 2048=\cdot 2259$ is considerably smaller than its expectation $3+3-3=3$, the degrees of freedom. The two sets of data may be regarded as homogeneous.

The variances of the estimates from the combined data are given by the diagonal elements of the matrix $\mathbf{T}^{-1}$.

$$
\begin{aligned}
& \mathrm{V}\left(\dot{y}_{1}\right)=\cdot 2275 / \mathrm{r} 743=\mathrm{IO}^{-3}(\cdot \mathrm{I} 30522) \\
& \mathrm{V}\left(\dot{y}_{2}\right)=\cdot 2379 / \mathrm{I} 743=\mathrm{IO}^{-3}(\cdot \mathrm{I} 36489) \\
& \mathrm{V}\left(\dot{y}_{3}\right)=\cdot 065 \mathrm{I} / \mathrm{1} 743=\mathrm{IO}^{-3}(\cdot 037349)
\end{aligned}
$$

These are only approximate values, the exact values being obtainable from the inverse of the information matrix calculated at the estimated values.

If Kosambi's formula is assumed, there are only two parameters $y_{1}$ and $y_{3}$ to be estimated. The appropriate scores and information matrix in this case are calculated from the values obtained before for $y_{1}, y_{2}$ and $y_{3}$ considering them as free parameters. The trial values of $y_{1}, y_{2}$ and $y_{3}$ are the same as before. To start with calculate

$$
\begin{array}{rlrl}
\mu_{1} & =\frac{\mathrm{I}-4 y_{3}^{2}}{\left(\mathrm{I}+4 y_{1} y_{3}\right)^{2}}, \quad \mu_{3} & =\frac{\mathrm{I}-4 y_{1}^{2}}{\left(\mathrm{I}+4 y_{1} y_{3}\right)^{2}} \\
& =\frac{.980400}{\mathrm{I} \cdot 205604}, & & =\frac{5 \mathrm{I} 0000}{\mathrm{I} \cdot 205604} \\
& =\cdot 8 \mathrm{I} 3202, & & =\cdot 423024 \\
\mu_{1}^{2} & =\cdot 66 \mathrm{I} 297, \mu_{1} \mu_{3} & =\cdot 344004, \quad \mu_{3}^{2}=\cdot \mathrm{I} 78949 .
\end{array}
$$

The efficient scores $\psi_{1}$ and $\psi_{3}$ are,

$$
\begin{aligned}
\psi_{1} & =\Phi_{1}+\mu_{1} \Phi_{2} \\
& =289 \cdot 8703 \mathrm{IO}+\cdot 813202(-273 \cdot 766048) \\
& =67 \cdot 2432 \mathrm{II} \\
\psi_{3} & =\Phi_{3}+\mu_{3} \Phi_{2} \\
& =173 \cdot 995492+\cdot 423024(-273 \cdot 766048) \\
& =58 \cdot 185880
\end{aligned}
$$


Information matrix for $\mathrm{y}_{1}, \mathrm{y}_{3}$.

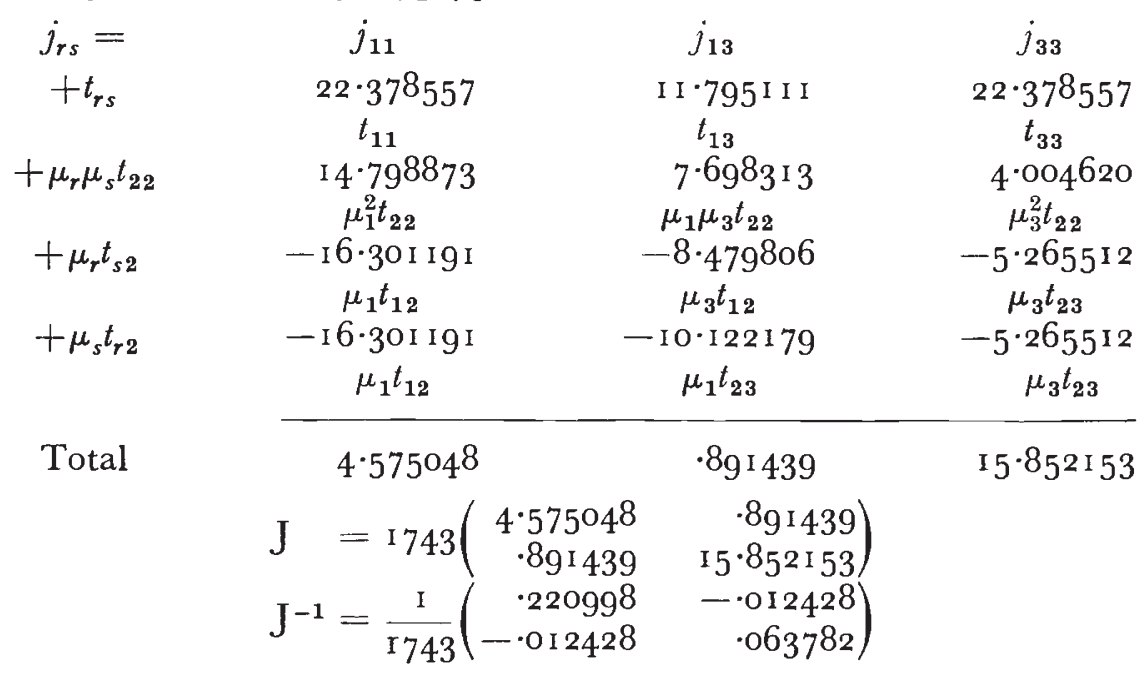

The additive corrections to $y_{1}$ and $y_{3}$ are given by

$$
\begin{aligned}
& d y_{1}=\frac{\mathrm{I}}{\mathrm{I} 743}\left[\cdot 220998(67 \cdot 2432 \text { I I })-\cdot 0 \mathrm{I} 2428\left(5^{8} \cdot \mathrm{I} 85880\right)\right] \\
& =\mathrm{I} 4 \cdot \mathrm{I} 3748 \mathrm{I} / \mathrm{I} 743=\cdot 008 \mathrm{II} \text { I } \\
& d y_{3}=\frac{\mathrm{I}}{\mathrm{I} 743}[-.0 \mathrm{I} 2428(\quad)+.063782( \\
& =2 \cdot 8755^{13} / 1743=\text { ooo } 64975
\end{aligned}
$$

so that the estimates of $y_{1}$ and $y_{3}$ are

$$
358 \text { I I I and } 070165
$$

and the estimate of $y_{2}$ as obtained from the formula is

$$
\begin{aligned}
\ddot{y}_{2} & =\left(\ddot{y}_{1}+\ddot{y}_{3}\right) /\left(\mathrm{I}+4 \ddot{y}_{1} \ddot{y}_{3}\right) \\
& =(\cdot 428276) / \mathrm{I} \cdot \mathrm{I} 00507=389 \mathrm{I} 62
\end{aligned}
$$

which differ very slightly from the estimates obtained by treating the $y$ 's as free parameters. The goodness of fit of Kosambi's formula to the cross over values indicated by the data may be tested as follows.

This needs the evaluation of the total efficient score $\ddot{\Phi}_{2}$ at these estimated values. As before, a good approximation to this value can be obtained by using the formula

$$
\begin{aligned}
& \ddot{\Phi}_{2}=\Phi_{2}-\mathrm{N}\left[i_{21}\left(\ddot{y}_{1}-\cdot 35\right)+i_{22}\left(\ddot{y}_{2}-39\right)+i_{23}\left(\ddot{y}_{3}-\cdot 07\right)\right. \\
& =-273 \cdot 766048-\mathrm{I} 743[-20 \cdot 045685(\cdot 008 \text { I I I }) \\
& \left.\quad+22 \cdot 378557\left(-\cdot 00083^{8}\right)-\text { I } 2 \cdot 4473 \text { I } 2(\cdot 000 \text { I } 64975)\right] \\
& =-273 \cdot 766048+3 \text { I } 9 \cdot 66 \text { I } 9 \text { I } 2 \\
& =\quad 45 \cdot 895864 \\
& \mathrm{I} / \mathrm{V}\left(\ddot{\Phi}_{2}\right)=\frac{\mathrm{I}}{\mathrm{N}}\left[i^{22}+\mu_{1}^{2} i^{11}+\mu_{3}^{2} i^{33}+2 \mu_{1} \mu_{3} i^{13}-2 \mu_{1} i^{12}-2 \mu_{3} i^{23}\right] \\
& =\mathrm{IO}^{-4}(\cdot 270694) .
\end{aligned}
$$


The quantity

$$
\begin{aligned}
\chi^{2}=\left(\ddot{\Phi}_{2}\right)^{2} / \mathrm{V}\left(\ddot{\Phi}_{2}\right) & =\left(45^{8995864}\right)^{2} \times \mathrm{Io}^{-4}(\cdot 270694) \\
& =\cdot 0570
\end{aligned}
$$

can be used as $\chi^{2}$ with one degree of freedom to test Kosambi's formula. The observed $\chi^{2}$ is very small, thus indicating fair agreement with Kosambi's formula.

The variances of the estimates obtained by using Kosambi's formula are,

$$
\begin{aligned}
& \mathrm{V}\left(\ddot{y}_{1}\right)=\frac{j^{11}}{\mathrm{~N}}=\cdot \frac{\cdot 220998}{\mathrm{I} 743}=\mathrm{IO}^{-3}(\cdot \mathrm{I} 2679 \mathrm{I}) \\
& \mathrm{V}\left(\ddot{y}_{3}\right)=\frac{j^{33}}{\mathrm{~N}}=\frac{\cdot 063782}{\mathrm{I} 743}=\mathrm{IO} \mathrm{O}^{-3}(\cdot 036593) \\
& \mathrm{V}\left(\ddot{y}_{2}\right)=\frac{\mu_{1}^{2} j^{11}+2 \mu_{1} \mu_{3} j^{13}+\mu_{3}^{2} j^{33}}{\mathrm{~N}}=\frac{\cdot \mathrm{I} 49008}{\mathrm{I} 743}=\mathrm{IO}-3(\cdot 085489) .
\end{aligned}
$$

By comparing these variances with those obtained before, we find the percentage increase in efficiency by using Kosambi's formula as

$$
2 \cdot 94,59 \cdot 66,2 \cdot 06
$$

for $y_{1}, y_{2}$ and $y_{3}$ respectively.

\section{THE INCREASE IN PRECISION BY THE USE OF EFFICIENT SCORES}

In the previous sections, methods have been developed for the appropriate scoring of data relating to the simultaneous segregation of three factors. It is, however, of importance to calculate the gain in efficiency by following this method instead of replacing the data by three marginal distributions obtained by ignoring one factor each time and treating the distributions as independent. In such a case the data can be scored by considering only one segment at a time. It is seen in section 3 that this makes no difference in the case of back crosses. The general investigation of this problem in the case of $\mathrm{F}_{2}$ intercrosses is difficult but an example may be considered to give an idea of the increase in efficiency.

Let the data consist of the results of the $F_{2}$ of $\frac{A b C}{a B c}$ classified in eight phenotypical classes. The variances and covariances of the estimates, from the data of the above type of $y_{1}, y_{2}, y_{3}$ considered as (I) free parameters and (2) subject to Kosambi's formula, can be calculated in each case by using $(a)$ the method of efficient scores and $(b)$ the individual segment method, and the relative efficiency of the method $(b)$ in each case can be found out. The following are the calculations in the particular case wherein the recombination fractions are

$$
y_{3}=.03, y_{1}=\cdot 28 \text { and } y_{2}=\cdot 30
$$


(a) Method of efficient scores taking y's as free parameters

Using table 4 , the appropriate S-functions for the triple heterozygote $\mathrm{AbC} / \mathrm{aBc}$ are calculated at the above chosen values.

$$
\begin{aligned}
& \mathrm{S}_{3}=\frac{1}{4}\left(y_{2}+y_{3}-y_{1}\right)=\text {. } 0125 \\
& \mathrm{~S}_{2}=\frac{1}{4}\left(2-y_{3}-y_{2}-y_{1}\right)=\cdot 3475 \\
& \mathrm{~S}_{1}=\frac{1}{4}\left(y_{2}+y_{1}-y_{3}\right)=\cdot 1375 \\
& \mathrm{~S}_{0}=\frac{1}{4}\left(y_{3}+y_{1}-y_{2}\right) \quad=\cdot 0025 \\
& \text {.500o (check) }
\end{aligned}
$$

The values of the $l$ 's, $m$ 's, $n$ 's of table 3 with I replaced by $\frac{1}{2}$ are

\begin{tabular}{|c|c|c|c|c|c|c|c|}
\hline \multirow{2}{*}{ Phenotype } & \multirow{2}{*}{$\begin{array}{c}\text { Probability } \\
\qquad 16 \pi\end{array}$} & \multicolumn{6}{|c|}{ Derivatives and scores } \\
\hline & & $16 \frac{\partial \pi}{\partial y_{1}}$ & $\frac{1}{\pi} \frac{\partial \pi}{\partial y_{1}}$ & $16 \frac{\partial \pi}{\partial y_{2}}$ & $\frac{1}{\pi} \frac{\partial \pi}{\partial y_{2}}$ & $16 \frac{\partial \pi}{\partial y_{3}}$ & $\frac{1}{\pi} \frac{\partial \pi}{\partial y_{3}}$ \\
\hline \multirow[t]{2}{*}{$\begin{array}{l}\mathrm{ABC} \\
\mathrm{AbC} \\
\mathrm{ABc} \\
\mathrm{Abc} \\
\mathrm{aBC} \\
\mathrm{abC} \\
\mathrm{aBc} \\
\mathrm{abc}\end{array}$} & $\begin{array}{r}6 \cdot 2771 \\
3 \cdot 6829 \\
1 \cdot 7265 \\
\cdot 3135 \\
2 \cdot 0365 \\
\cdot 0035 \\
1 \cdot 9599 \\
\cdot 0001\end{array}$ & $\begin{array}{r}2 \cdot 22 \\
-2 \cdot 22 \\
-2 \cdot 22 \\
2 \cdot 22 \\
\cdot 02 \\
-\cdot 02 \\
-\cdot 02 \\
\cdot 02\end{array}$ & \multirow[t]{2}{*}{$\begin{array}{r}.353666 \\
-.602788 \\
-1 \cdot 285838 \\
7 \cdot 081338 \\
\cdot 009820 \\
-5 \cdot 714286 \\
-\cdot 010204 \\
200 \cdot 000000\end{array}$} & $\begin{array}{r}-6 \cdot 3^{8} \\
-\cdot 02 \\
6 \cdot 3^{8} \\
\cdot 02 \\
6 \cdot 3^{8} \\
\cdot 02 \\
-6 \cdot 3^{8} \\
-\cdot 02\end{array}$ & $\begin{array}{r}-.888944 \\
-.005430 \\
3.231970 \\
.637960 \\
2.739920 \\
5.714286 \\
-2.847084 \\
-200 \cdot 000000\end{array}$ & $\begin{array}{r}\cdot 22 \\
-\cdot 22 \\
\cdot 02 \\
-\cdot 02 \\
-\cdot 22 \\
.22 \\
-.02 \\
.02\end{array}$ & \multirow[t]{2}{*}{$\begin{array}{r}\cdot 025048 \\
-.029868 \\
\cdot 011584 \\
-.637960 \\
-.108028 \\
62.857140 \\
-.018204 \\
200 \cdot 000000\end{array}$} \\
\hline & I $6 \cdot 0000$ & $0 \cdot 00$ & & $0 \cdot 00$ & & $0 \cdot 00$ & \\
\hline
\end{tabular}

$$
\begin{aligned}
& l_{2}=l_{3}=m_{2}=m_{0}=n_{2}=n_{1}=-\frac{1}{2} \\
& l_{1}=l_{0}=m_{1}=m_{3}=n_{0}=n_{3}=\frac{1}{2}
\end{aligned}
$$

The probabilities, derivatives and scores are given in table 7 .

TABLE 7

Probabilities and scores

The information matrix i, per single observation, can be calculated by using the formulæ of section $3(b)$. Thus

$$
\begin{aligned}
& i_{11}=\frac{\mathrm{I}}{\mathrm{I} 6}[2 \cdot 22(\cdot 353666)+\ldots+\cdot 02(200 \cdot 000000)]=\mathrm{I} \cdot 55082 \mathrm{I}, \\
& i_{12}=\frac{\mathrm{I}}{\mathrm{I} 6}[2 \cdot 22(-.888944)+\ldots+\cdot 02(-200 \cdot 000000)]=-.8 \mathrm{I} 233 \mathrm{I},
\end{aligned}
$$

etc.

$$
\mathbf{i}=\left(\begin{array}{rrr}
\mathrm{I} \cdot 55082 \mathrm{I} & -\cdot 8 \mathrm{I} 233 \mathrm{I} & \text {-I } 73998 \\
-\cdot 8 \mathrm{I} 233 \mathrm{I} & 3 \cdot 64289 \mathrm{I} & -\cdot 2 \mathrm{I} 3733 \\
\cdot \mathrm{I} 73998 & -\cdot 2 \mathrm{I} 3733 & \text { I.II I I I }
\end{array}\right)
$$

The covariance matrix $v$, per single observation, of the maximum likelihood estimates obtained by choosing such values of $y_{1}, y_{2}, y_{3}$ 
which make the scores for an observed set of frequencies vanish, is the inverse of the information matrix.

$$
\mathbf{v}=\left(\begin{array}{rrr}
\cdot 737882 & \cdot r 59589 & -\cdot 08439 \mathrm{r} \\
\cdot 159589 & \cdot 312 \mathrm{r} 39 & \cdot 03486 \mathrm{r} \\
-.08439 \mathrm{r} & \cdot 03486 \mathrm{r} & \cdot 914923
\end{array}\right)
$$

(b) Method of efficient scores: y's being subject to Kosambi's formula

The information matrix $\mathbf{j}$, per single observation, for $y_{1}, y_{3}$ in this case is obtained from i, calculated above, by using the formula given in section 5 and illustrated in section 6 .

The procedure of computation is as follows :-

$\begin{array}{cccc} & \mu_{1}=.93267 \mathrm{I} & , \mu_{3}=\cdot 642498 & \\ j_{r s} & j_{11} & j_{13} & j_{33} \\ i_{r s} & \mathrm{I} \cdot 55082 \mathrm{I} & \cdot \mathrm{r} 73998 & \mathrm{I} \cdot 1 \mathrm{I} 7 \mathrm{I} 8 \mathrm{I} \\ \mu_{r} \mu_{s} i_{22} & 3 \cdot 168852 & 2 \cdot 182996 & \mathrm{I} \cdot 503807 \\ \mu_{r} i_{s 2} & -\cdot 757637 & -.52192 \mathrm{I} & -\cdot 137323 \\ \mu_{s} i_{2 r} & -\cdot 757637 & -\cdot 199342 & -\cdot 137323 \\ \text { Total } & 3.204399 & \mathrm{I} \cdot 63570 \mathrm{I} & 2 \cdot 346342\end{array}$

The covariance matrix $u$, per single observation, of the estimates of $y_{1}$ and $y_{3}$ is the inverse of $\mathbf{j}$.

$$
\mathbf{u}=\left(\begin{array}{rr}
\cdot 48447 \mathrm{r} & -.33773^{8} \\
-.33773^{8} & .66 \mathrm{r} 64^{2}
\end{array}\right)
$$

If $\ddot{y}_{1}$ and $\ddot{y}_{3}$ are the estimates of $y_{1}$ and $y_{3}$ then, substituting these values in Kosambi's formula, we get $\ddot{y}_{2}$, the estimate of $y_{2}$. The variance of $\ddot{y}_{2}$ is calculated by the formula,

$$
\begin{aligned}
& \mathrm{V}\left(\ddot{y}_{2}\right)=\cdot 48447 \mathrm{r}\left(\cdot 93^{267} \mathrm{r}\right)^{2}-2(\cdot 337738)(\cdot 93267 \mathrm{r})(\cdot 642498) \\
& =\cdot 289786 \text { per single observation. } \\
& +\cdot 661642(\cdot 642498)^{2}
\end{aligned}
$$

\section{(c) Individual segment method taking $y$ 's as free}

In the individual segment method, only two factors are considered. each time and the data are scored for the corresponding recombination fraction. The results of the cross $\mathrm{AbC} \times \mathrm{aBc}$ supply data in repulsion for the estimation of $y_{3}$ and $y_{1}$ and in coupling for $y_{2}$. To score for $y_{1}$, it is necessary to consider the scores appropriate to data in repulsion as given in Mather's book, The Measurement of Linkage in Heredity, p. 6I (first edition). The observed frequencies in the eight phenotypical classes are represented by $n_{i j k}$ as in table 2. Ignoring the classification with respect to the first factor we get the scores at $y_{1}=\cdot_{2} 8$ 
in the four phenotypical classes and observed frequencies as given in table 8 .

TABLE 8

Scores in repulsion at $\mathrm{y}_{1}=\cdot 28$

$\begin{array}{ccc}\text { Phenotype } & \begin{array}{c}\text { Observed frequency, ignoring } \\ \text { the first factor }\end{array} & \text { Scores } \\ \text { BC } & n_{111}+n_{011} & -269438 \\ \text { Bc } & n_{110}+n_{010} & -\cdot 607639 \\ \text { bC } & n_{101}+n_{001} & -\cdot 607639 \\ \text { bc } & n_{100}+n_{000} & 7 \cdot 142857\end{array}$

If the total score for $y_{1}$ by this method is represented by $Q_{1}$, then $Q_{1}=\cdot 269438\left(n_{111}+n_{011}\right)-\cdot 607639\left(n_{110}+n_{010}+n_{101}+n_{001}\right)$

The scores for $y_{2}$ and $y_{3}$ are similarly calculated. $+7 \cdot 142857\left(n_{100}+n_{000}\right)$

$$
\begin{aligned}
& \mathrm{Q}_{2}=-\cdot{ }^{5} 5_{2249}\left(n_{111}+n_{101}\right)+2 \cdot 745098\left(n_{110}+n_{100}+n_{011}+n_{001}\right)
\end{aligned}
$$

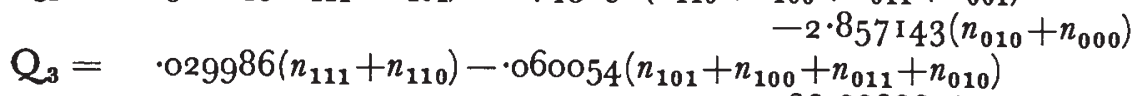

$$
\begin{aligned}
& +66 \cdot 666667\left(n_{001}+n_{000}\right)
\end{aligned}
$$

The estimate of $y_{i}$ is found, by this method, by choosing that value of $y_{i}$ which makes the score $Q_{i}$ zero for the observed set of frequencies. The estimates differ from the true values unless the frequencies are the same as their expected values. If the probabilities of given departures of the Q's from their expected values or simply their sampling errors in large samples are known, then the corresponding errors introduced in the estimate can be calculated. Since the Q's are linear functions of the frequencies in the eight phenotypical classes with probabilities as given in table 7 , the covariance $m_{i j}$, per single observation, between $Q_{i}$ and $Q_{j}$ can be calculated (as given in Fisher's Statistical Methods, p. 303, ninth edition) by taking the triple products of coefficients of the frequency for any class in $Q_{i}$ and $Q_{j}$ and the probability for that class and summing over all the eight classes. Thus

$$
\begin{aligned}
m_{11} & =\frac{\mathrm{I}}{\mathrm{I} 6}\left[(\cdot 269438)^{2}(6 \cdot 277 \mathrm{I}+2 \cdot 0365)+(\cdot 607639)^{2}(\mathrm{I} \cdot 7265+\mathrm{I} \cdot 9599\right. \\
& \left.=\mathrm{I} \cdot 20786 \mathrm{o} . \quad+3 \cdot 6829+\cdot 0035)+(7 \cdot 142857)^{2}(\cdot 3 \mathrm{I} 35+\cdot 000 \mathrm{I})\right]
\end{aligned}
$$

The matrix $\mathrm{m}=\left(m_{i j}\right)$, thus calculated, comes out as

$$
\mathrm{m}=\left(\begin{array}{rrr}
\mathrm{r} \cdot 270860 & 526986 & -.002269 \\
\cdot 526986 & 3 \cdot \mathrm{I} 18356 & .046039 \\
-.002269 & \cdot 046039 & \mathrm{r} \cdot 002248
\end{array}\right)
$$

The covariance of the estimates of $y_{i}$ and $y_{j}$ is given by $m_{i j} / m_{i i} m_{j j}$ 
so that, using the above elements, we can set up the covariance matrix $\boldsymbol{v} *$ of the estimates of $y_{1}, y_{2}, y_{3}$.

$$
\mathbf{v} *=\left(\begin{array}{rrr}
\cdot 827907 & \cdot \text { I } 399 \text { I } 3 & -\cdot 001874 \\
\cdot \text { I } 39913 & \cdot 320680 & \cdot 01473 \text { I } \\
-\cdot 001874 & \cdot 01473 \text { I } & \cdot 997804
\end{array}\right)
$$

(d) Individual segment method, $y$ 's being subject to Kosambi's formula

In this there are two parameters, $y_{1}$ and $y_{3}$, to be estimated. The scores $\mathrm{P}_{1}, \mathrm{P}_{3}$ for $y_{1}$ and $y_{3}$ by this method are,

$$
\begin{aligned}
& \mathrm{P}_{1}=\mathrm{Q}_{1}+\mu_{1} \mathrm{Q}_{2} \\
& \mathrm{P}_{3}=\mathrm{Q}_{3}+\mu_{3} \mathrm{Q}_{2}
\end{aligned}
$$

so that, knowing $Q_{1}, Q_{2}, Q_{3}$ considered above, and the differential coefficients $\mu_{1}, \mu_{3}$, the P's can be calculated. As the covariances of the Q's are known the covariances of P's can be calculated as follows

$$
\begin{aligned}
\mathrm{V}\left(\mathrm{P}_{1}\right) & =\mathrm{V}\left(\mathrm{Q}_{1}\right)+\mu_{1}^{2} \mathrm{~V}\left(\mathrm{Q}_{2}\right)+2 \mu_{1} \operatorname{Cov}\left(\mathrm{Q}_{1} \mathrm{Q}_{2}\right) \\
& =\mathrm{I} \cdot 207860+(\cdot 93267 \mathrm{I})^{2}\left(3 \cdot \mathrm{I} \mathrm{I} 835^{6}\right)+2(\cdot 93267 \mathrm{I})(\cdot 526986) \\
& =4 \cdot 90343^{8} . \\
\operatorname{Cov}\left(\mathrm{P}_{1} \mathrm{P}_{3}\right) & =\operatorname{Cov}\left(\mathrm{Q}_{1} \mathrm{Q}_{3}\right)+\mu_{1} \operatorname{Cov}\left(\mathrm{Q}_{2} \mathrm{Q}_{3}\right)+\mu_{3} \operatorname{Cov}\left(\mathrm{Q}_{1} \mathrm{Q}_{2}\right) \\
& +\mu_{1} \mu_{3} \mathrm{~V}\left(\mathrm{Q}_{2}\right) \\
& =2 \cdot 247897 .
\end{aligned}
$$

Similarly

$$
\mathrm{V}\left(\mathrm{P}_{3}\right)=2 \cdot 348684
$$

Thus $d$, the covariance matrix, per single observation, of $P_{1}, P_{3}$ is

$$
\mathbf{d}=\left(\begin{array}{ll}
4 \cdot 903438 & 2 \cdot 247897 \\
2 \cdot 247897 & 2 \cdot 348684
\end{array}\right)
$$

If the Q's are independent, i.e. if three sets of data containing the same number of observations as the total in the case were independently observed for the three segments separately, then the individual segment method is the most efficient method. In this case the covariance matrix a, per single observation, of $P_{1}, P_{3}$ is obtained from the above calculations by dropping the covariance terms. Thus

and

$$
\begin{aligned}
\mathrm{V}\left(\mathrm{P}_{1}\right) & =\mathrm{I} \cdot 207860+(\cdot 93267 \mathrm{I})^{2}(3 \cdot \mathrm{I} \text { I } 8356) \\
& =3 \cdot 92043^{\circ}
\end{aligned}
$$

$$
a=\left(\begin{array}{ll}
3 \cdot 920430 & \mathrm{I} \cdot 868640 \\
\mathrm{I} \cdot 868640 & 2 \cdot 289524
\end{array}\right)
$$

Its reciprocal $\mathbf{a}^{-1}$ gives, when the $Q$ 's are independent, the covariance matrix of the estimates obtained by choosing values of $y_{1}$ and $y_{3}$ which make the scores $P_{1}$ and $P_{3}$ vanish. This, on calculation, comes out as

$$
\mathbf{a}^{-1}=\left(\begin{array}{rr}
\cdot 417483 & -.340737 \\
-.340737 & \cdot 714871
\end{array}\right)
$$


When the Q's are not independent the estimates, obtained by choosing such values of $y_{1}$ and $y_{3}$ which make the scores $\mathrm{P}_{1}$ and $\mathbf{P}_{3}$ for the observed set of frequencies vanish, have the covariance matrix $u_{*}$ given by

$$
\mathrm{u}^{*}=\mathrm{a}^{-1} \mathrm{da}^{-1}
$$

This is a triple product of the matrices $a^{-1}, d$ and $a^{-1}$. To evaluate this the product $\mathrm{a}^{-1} \mathbf{d}$ may be found first and then multiplied by $\mathrm{a}^{-1}$. The method of multiplying two matrices is to construct a matrix whose element in the $i$-th row and the $j$-th column is the sum of products of the ordered elements in the $i$-th row of the first matrix and $j$-th column of the second matrix.

$$
\begin{aligned}
\mathbf{u} * & =\left(\begin{array}{rr}
\cdot 4 \mathrm{I} 7483 & -\cdot 340737 \\
-\cdot 340737 & \cdot 7 \mathrm{I} 487 \mathrm{I}
\end{array}\right)\left(\begin{array}{rr}
4 \cdot 903438 & 2 \cdot 247897 \\
2 \cdot 247897 & 2 \cdot 348684
\end{array}\right)^{\mathrm{a}^{-1}} \\
& =\left(\begin{array}{rr}
\mathrm{I} \cdot 28 \mathrm{II} 6 \mathrm{O} & \cdot \mathrm{I} 38 \mathrm{I} 75 \\
-\cdot 063827 & \cdot 9 \mathrm{I} 3064
\end{array}\right)\left(\begin{array}{rr}
\cdot 4 \mathrm{I} 7483 & -\cdot 340737 \\
-\cdot 340737 & \cdot 7 \mathrm{I} 487 \mathrm{I}
\end{array}\right) \\
& =\left(\begin{array}{rr}
-48778 \mathrm{I} & -\cdot 33776 \mathrm{I} \\
-\cdot 33776 \mathrm{I} & \cdot 674472
\end{array}\right)
\end{aligned}
$$

The variance, per single observation, of the estimate of $y_{2}$ as calculated from the formula given in section $7(b)$ is

$$
\begin{aligned}
(\cdot 48778 \mathrm{I})(\cdot 93267 \mathrm{I})^{2}-2(\cdot 33776 \mathrm{I})(\cdot 93267 \mathrm{I})(\cdot 642498) & +(\cdot 674472)(\cdot 642498)^{2}=\cdot 297933
\end{aligned}
$$

A comparison is made below of the covariance matrices $\mathbf{v}, \mathbf{v}$,

\begin{tabular}{|c|c|c|c|c|c|c|c|c|c|}
\hline \multirow{3}{*}{ Method } & \multicolumn{6}{|c|}{ Variances of estimates of $y$ 's treated as } & \multirow{2}{*}{\multicolumn{3}{|c|}{$\begin{array}{l}\text { Percentage } \\
\text { increase in } \\
\text { efficiency due } \\
\text { to Kosambi's } \\
\text { formula }\end{array}$}} \\
\hline & \multicolumn{3}{|c|}{ Free parameters } & \multicolumn{3}{|c|}{$\begin{array}{l}\text { Subject to Kosambi's } \\
\text { formula }\end{array}$} & & & \\
\hline & $y_{1}$ & $y_{2}$ & $y_{3}$ & $y_{2}$ & $y_{2}$ & $y_{3}$ & $y_{1}$ & $y_{2}$ & $y_{3}$ \\
\hline $\begin{array}{l}\text { 2) Efficient scores } \\
\text { 3) Individual segment }\end{array}$ & $\begin{array}{l}.737882 \\
.827907 \\
\end{array}$ & $\begin{array}{r}312139 \\
.320680 \\
\end{array}$ & $\begin{array}{r}.914923 \\
.997804 \\
\end{array}$ & $\begin{array}{r}484471 \\
\cdot 487781 \\
\end{array}$ & $\begin{array}{l}.289786 \\
.297933 \\
\end{array}$ & $\begin{array}{l}.661642 \\
.674472 \\
\end{array}$ & $\begin{array}{l}52 \cdot 30 \\
69 \cdot 73\end{array}$ & $\begin{array}{l}7 \cdot 71 \\
7 \cdot 63\end{array}$ & $\begin{array}{l}38 \cdot 2 i \\
47 \cdot 9\end{array}$ \\
\hline $\begin{array}{l}\text { 1) Relative efficiency } \\
=\frac{(a)}{(\beta)} \times 100\end{array}$ & $89 \cdot 12$ & $97 \cdot 34$ & $9^{1} \cdot 69$ & $99 \cdot 32$ & $97 \cdot 3^{1}$ & $9^{8 \cdot 10}$ & $\cdots$ & $\cdots$ & ". \\
\hline
\end{tabular}
$\mathbf{u}, \mathbf{u} *$ of the estimates obtained by the four methods discussed above. Choosing the variances alone we have the comparisons as shown in table 9 .

TABLE 9

Variances of estimates and relative efficiencies

A measure of overall efficiency can be obtained by comparing the covariance matrices of the estimates by the two methods. Instead 
of the simple ratio of the determinants, its $s$-th root, where $s$ is the number of parameters estimated, may be defined as the relative efficiency of one method as compared with another. Thus, when $y$ 's are considered as free parameters the overall efficiency of the individual segment method is

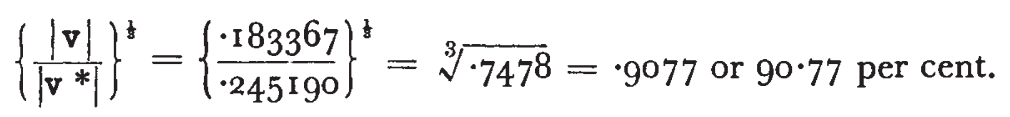

The corresponding efficiency when $y$ 's are subject to Kosambi's formula is

$$
\left\{\frac{|\mathrm{u}|}{|\mathrm{u} *|}\right\}^{\frac{1}{2}}=\cdot 980 \mathrm{I} \text { or } 98 \cdot 0 \text { I per cent. }
$$

The overall increase in efficiency by using Kosambi's formula can be calculated by comparing the covariance determinants of the estimates of $y_{1}$ and $y_{3}$ alone. This increase for the method of efficient scores is

$$
\frac{\left|\begin{array}{ll}
\cdot 737882 & \cdot I 73998 \\
\cdot \mathrm{I} 73998 & \cdot 9 \mathrm{I} 4923
\end{array}\right|}{|\mathrm{u}|^{\frac{1}{2}}}-\mathrm{I}=79 \cdot 89 \text { per cent. }
$$

and for the individual segment method

$$
\frac{\left|\begin{array}{rr}
.827909 & -.001874 \\
-.001874 & .997804
\end{array}\right|}{\left|u^{\frac{1}{2}} *\right|^{\frac{1}{2}}}-\mathrm{I}=96.06 \text { per cent. }
$$

These calculations lead to the following conclusions, (I) The use of Kosambi's formula, when applicable, considerably increases the efficiency of the estimates by either method. In the particular example chosen the overall increase in efficiency is 79.89 per cent. for the most efficient method and $96 \cdot 06$ per cent. for the individual segment method. (2) The loss of efficiency due to the simpler analysis of the individual segment method is smaller when the recombination fractions are estimated with the use of Kosambi's formula. In the above example the overall efficiency of the individual segment method, when $y$ 's are considered as free parameters, is 90.77 per cent. which increases to $98 \cdot 0$ r per cent. when $y$ 's are subject to Kosambi's formula.

\section{SUMMARY}

The following results have been discussed in this article.

I. The appropriate scoring of data giving the simultaneous segregation of three factors and the method of arriving at the best estimates of recombination fractions from data relating to various sources and types of crosses have been discussed in the two cases (i) when they are taken as free parameters and (ii) when they conform to Kosambi's formula. It has been observed that the scores and information matrix 
in the latter case are connected with those in the former by simple relations and in any practical example it is convenient to score the data considering the recombination fractions as free parameters and then deduce the total scores appropriate to the latter case.

It has been found, in the examples discussed in the article, that the estimates found by using Kosambi's formula have considerably smaller variances. This is so, for when this formula is true there are only two parameters to be estimated (the third one being deduced from the formula) and any method of estimation which does not make use of the formula, being different from the maximum likelihood method appropriate to the two parameters, is bound to be inefficient.

The use of such empirical relations as the one considered above among the parameters to be estimated, when known, enhances the precision of the estimates although they may not be strictly accurate. The assumption of a slightly inaccurate relationship may introduce bias in the estimates but such estimates are more useful than the less efficient estimates so long as the bias, in any case, is small in comparison with its standard error. This, in some way, is secured when the test for a hypothesis specifying some restrictions indicates close agreement with the observations. Kosambi's formula is very useful from this point of view, as its use considerably enhances the precision of the estimates. A test has been proposed to judge the validity of this formula in any particular case.

2. In view of the slightly heavier computation involved in the method of efficient scores an investigation has been made to find out the loss in efficiency due to the simpler method of scoring by considering the data as classified with respect to only two factors each time and considering them as independent distributions. The latter method is called the individual segment method.

It has been found that if the data consist of only back crosses both the methods lead to identical estimates, when the recombination fractions are considered as free parameters. This, however, is not true when the estimates are found subject to Kosambi's formula but the loss in efficiency is not expected to be considerable.

In the case of intercrosses a particular example has been chosen to find the relative efficiency of this method in the two cases when the recombination fractions are considered as (i) free parameters and (ii) subject to Kosambi's formula. It is found that the loss of information due to the simpler analysis is negligible when the recombination fractions conform to Kosambi's formula. This may not be generally true, but the loss in any case is not expected to be considerable. Similar results may be expected in the case of data giving the simultaneous segregation of more than three factors. Their exact treatment involves some complications because some extraneous parameters have to be estimated and accounted for in the evaluation of the variances of the estimates of the recombination fractions. This, however, awaits further study. 
Finally, I should like to thank Professor R. A. Fisher for his guidance and criticism during the preparation of this paper and $\mathrm{Dr} \mathrm{K}$. Mather for his valuable suggestions which led to a clarification of the various steps involved in this article.

\section{REFERENCES}

BHAT, N. R. 1948 .

An improved genetical map of Punnett's B-chromosome in the sweet pea Lathyrus odoratus $\mathrm{L}$.

7. Genet., 48, 3, 343-358.

DE WINTON, D., AND hALDANE, J. B. S. 1935 .

The genetics of Primula sinensis. III. Linkage in the diploid.

7. Genet., 31, 67-1 00 .

FISHER, R. A. 1935 .

The detection of linkage with dominant abnormalities.

Ann. Eugen., 6, 187-201.

FISHER, R. A. 1946.

A system of scoring linkage data with special reference to pied factors in mice.

Amer. Nat., 8o, 568-578.

KOSAMBI, D. D. $\quad$ I 944 .

The estimation of map distance from recombination values.

Ann. Eugen., 12, 172-1 76.

RAO, C. R. $\quad 1947$.

On minimum variance and the estimation of several parameters.

Proc. Camb. phil. Soc., 43, 280-283.

RAO, C. R. 1948.

Large sample tests of statistical hypotheses concerning several parameters with applications to problems of estimation.

Proc. Camb. phil. Soc., 44, 50-57. 